\title{
Simulating Reach Motions
}

\author{
Don B. Chaffin \\ Julian Faraway \\ University of Michigan \\ Xudong Zhang \\ Wright State University
}

Copyright (C) 1999 Society of Automotive Engineers, Inc.

\section{ABSTRACT}

Modeling normal human reach behavior is dependent on many factors. Anthropometry, age, gender, joint mobility and muscle strength are a few such factors related to the individual being modeled. Reach locations, seat configurations, and tool weights are a few other task factors that can affect dynamic reach postures.

This paper describes how two different modeling approaches are being used in the University of Michigan Human Motion Simulation Laboratory to predict normal seated reaching motions. One type of model uses an inverse kinematic structure with an optimization procedure that minimizes the weighted sum of the instantaneous velocity of each body segment. The second model employs a new functional regression technique to fit polynomial equations to the angular displacements of each body segment.

To develop and validate these models, 38 subjects of widely varying age and anthropometry were asked to perform reaching motions while seated in simulated vehicle or industrial workplace. Between 48 and 72 reaches were required of each subject, for a total of over 7000 reaches. During these motions a Qualysis motion capture system was combined with an Ascension Technology Corporation Flock of Birds system to record the movements of each person's torso, shoulders, arm, forearm and hand.

The paper discusses how the models differ in construction and performance, and how our existing biomechanical models can be linked to the new kinematic models for improved dynamic ergonomic evaluations.
Ongoing research being conducted on human motion simulations at the University of Michigan's HUMOSIM Laboratory also is described.

\section{INTRODUCTION}

1.1 THE GENERAL PROBLEM OF REACHING - When a person reaches to a specific location which we will refer to as a "target," as long as the target is within a comfortable distance the resulting hand movements will exhibit the following general attributes [1]

1. The trajectory, if unobstructed, will be nearly straight.

2. The velocity profile will be bell shaped.

3. The acceleration profile will have two peaks (i.e., accelerate and decelerate).

With repetition the absolute values for the kinematic parameters describing the hand motions will become more consistent, but the general features described above will not change. Any mass added to the hand has a tendency to reduce the velocity and acceleration values. What also has been documented is that the trajectories, velocities and accelerations of individual joints are not as simply defined as the hand trajectories, and may even exhibit reversals in direction during a simple hand motion. These observations date back to studies by Nikolai Bernstein in the 1920s of a blacksmith that showed very consistent and simple hammer tip trajectories, but much more complex and variable limb joint movements. This led to the famous "Bernstein's Problem" of how the human body overcomes an excessive number of degrees of freedom to guide the hand or foot to a specific target [2]. Many theories and experiments have been conducted to develop an answer to the Bernstein Problem over the last 70 years, but as Gielen et al. [2] state:

"In summary, progress in understanding the control of the large number of available degrees of freedom is somewhat disappointing despite the large increase of experimental data since Bernstein." 
Since a fundamental neurophysiological understanding of reaching behavior has eluded us so far, have we progressed enough to provide useful reach simulations? What follows is a review of several approaches to this complex problem.

\subsection{TRADITIONAL MODELS FOR REACHING}

SIMULATION - Bernstein's notion of hand motion control centered on the concept of "functional synergy," that the neuromuscular and skeletal components become organized into highly structured, task specific ensembles. These ensembles provide motor control templates or motor memories that are stored in the motor cortex of the brain. These templates contain variables about the world (i.e., space, time and forces involved) to allow a task to be categorized, as well as control parameters, which direct the coordination of muscle actions [1]. In this latter regard, it must be remembered that individual muscle force and displacement control is mediated to a large extent by the stretch rate sensitive muscle spindles that monitor muscle displacement. This means that feedback is always present to assure a specific higher level command is resulting in the desired movement of the hand. This realization has produced adaptive feedforward models, a simple version of which is shown diagrammatically in Figure 1.

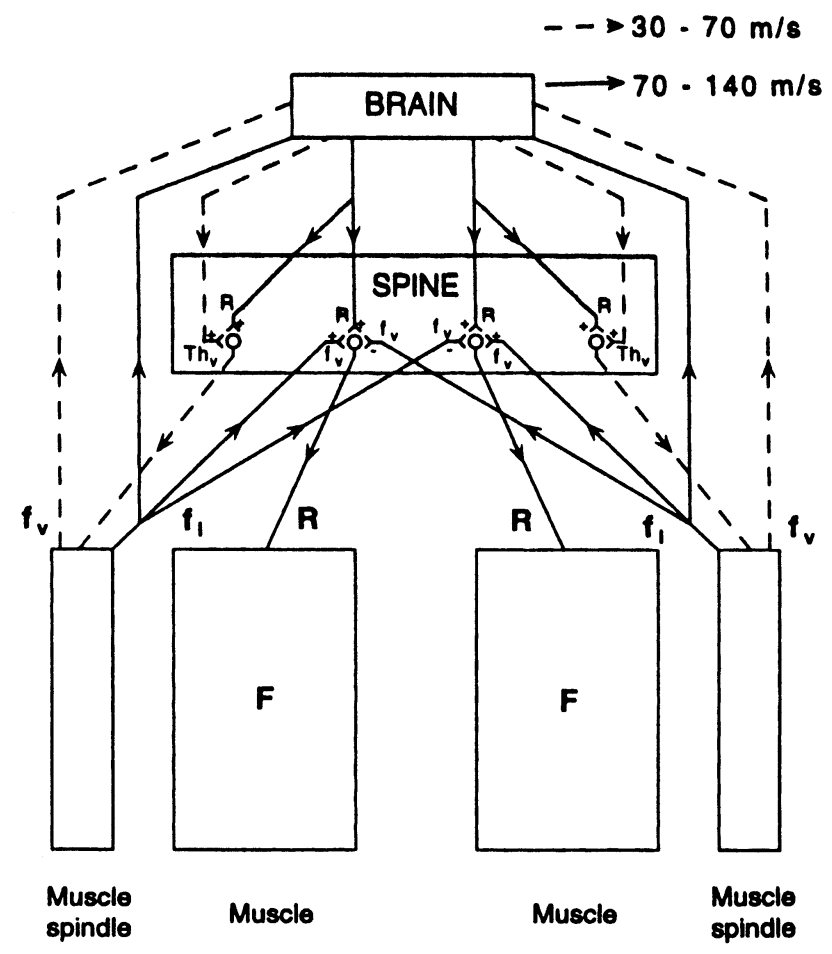

Figure 1. Adaptive feed forward model of muscle control wherein $F$ and $R$ are force and activation vectors [3].

Two things should be noted in Figure 1. First is the geometric simplicity, i.e., only two muscles. This would be acceptable for a single joint, co-planar motion simulation, but as noted earlier, multiple joints and degrees of freedom must be monitored and controlled during even a simple hand motion, and thus the two- muscle model displayed is overly simplistic. Secondly, muscle spindle feedback is shown with delay loops of 70$140 \mathrm{~ms}$. This means that only about 10 simple adjustments could be expected, on average, during a one second hand motion. Also, other feedback from Golgi tendon organs, Pacinian sensory organs, the vestibular system and other force and motion sensitive systems participate in any body motion, thus further complicating the neurophysiological basis for motion simulation.

This has led to other approaches, which have been evaluated for a well established movement pattern, mainly walking. One approach is to use forward dynamics, wherein a specific cost function or performance criterion (e.g., sum of muscle forces, or energy efficiency, or positional control) is minimized in a linear or non-linear dynamic optimization algorithm [4,5]. In this approach the problem of redundant motor systems, such as the control provided by the muscle spindle system $\lambda$ feedback loop and the direct cortico-spinal commands shown in Figure 1, must be resolved. Feldman and Levin [6], proposed that such redundancy in multi-joint motion systems could be solved by setting a hierarchy to the thresholds for which certain muscle activation commands would be accepted. Unfortunately experimental evidence has not disclosed the best hierarchical threshold scheme.

One of the major challenges in motion simulation is to develop a solution to the problem of simultaneous movement goals. Optimization methods work best when a single criterion is being maximized or minimized. Computationally fast and robust algorithms are available to assist in developing solutions for these types of problems. But take the case when a person is reaching far out to the side. To do so he or she must shift their weight, possibly lifting and moving the contra-lateral leg away from the target to maintain balance at the same time the upper torso and upper extremity are moved towards the target. Thus two motion tasks are performed simultaneously. In this situation, the primary task is to reach towards the target, but the secondary task is to maintain balance while on one foot. This maneuver has been described as shown in Figure 2 from Massion et al [7].

Evidence that a person can solve this difficult problem quickly comes from movement capture studies which clearly show that a person uses "Anticipator Posture Control." Before the center-of-gravity is outside the person's balance frame, the contra-lateral leg and lower torso begin to move in an opposite direction from the upper torso and hand (i.e., one anticipates that they will be out of balance), thus assuring dynamic balance is maintained. 


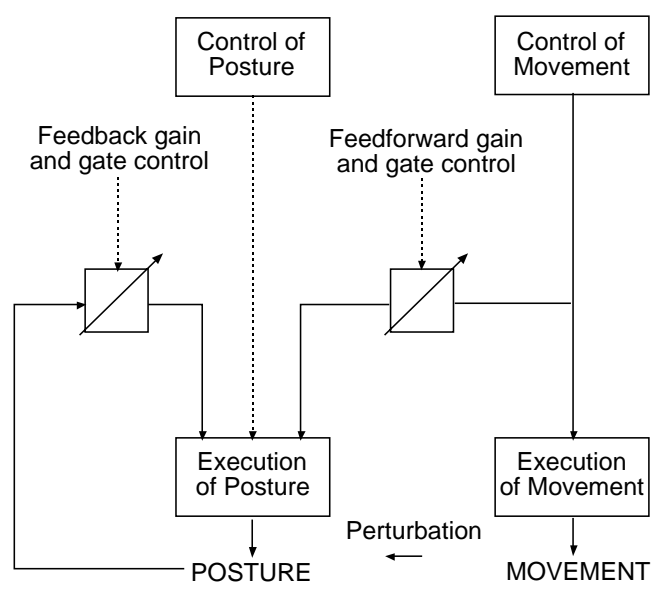

Figure 2. Feedforward and feedback adjustment of posture. The diagram represents the two mechanisms involved in compensating for a postural perturbation. The central control of posture is indicated by a dotted line. Two phasic mechanisms minimize the postural

disturbance. They operate through a feedback loop and a feedforward control. The feedforward control acts through internal collaterals from the movement control pathways on an adaptive network involved in postural control. Both mechanisms are under adaptive gate and gain control [7].

Because of the extreme complexity of such multijoint movements, models based on control theory have only provided one approach to understanding coordinated motions. Over the past decade Artificial Neural Networks or ANNs have been developed to predict patterns of muscle activity necessary to perform a given motion or exertion. ANNs provide a structured empirical method of identifying these patterns by allowing an investigator to hypothesize an organization of neurons and muscles, and then ask the question: What level of specific neuromuscular activation would result in a desired reaction, i.e. to move the hand along a specific trajectory, or create a desired force or moment at a specific joint? The complexity of the network that one would use depends on the number of neural nodes and muscle actions believed necessary to accurately model an activity. A very simple network is depicted in Figure 3, which would allow several different inputs $X_{p}$ to have a weighted $W_{k p}$ summation effect on an output $Y_{k}$.

The technical challenge in ANN development is to derive the appropriate weighting values $\mathrm{W}_{k}$. To do so requires that a set of data be used wherein a large variety of input and output conditions have been studied. These data are used to "train" the network (i.e., develop a set of $W_{k}$ values) by systematically changing the model $W_{k}$ values and comparing the output values to the data for given input values. Once a given level of accuracy is achieved, the model can be used to predict output

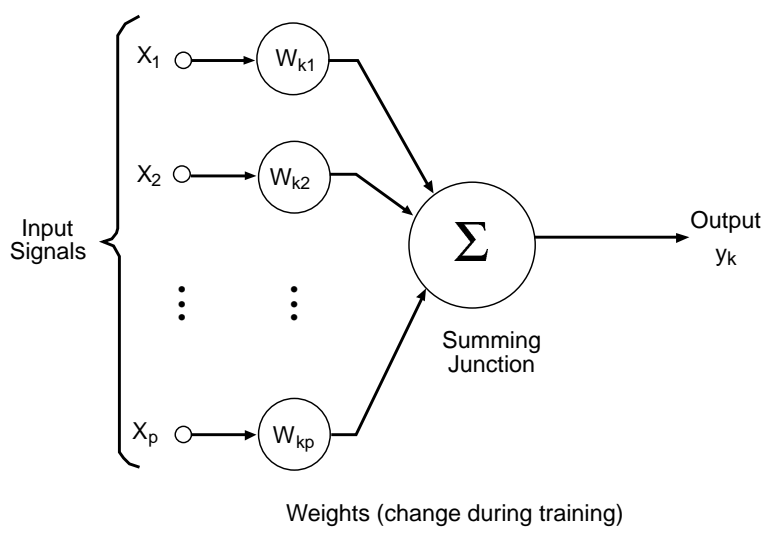

Figure 3. A simple ANN used to model the effect of several different inputs on an output.

performance for a more general set of input conditions, i.e., it can be used to simulate performance. For locomotion, ANNs have used muscle EMG amplitudes as input patterns to predict desired joint motion patterns, as described by Vaughan [8]. For torso exertion modeling ANNs were developed that related movement input loads to muscle EMG levels by Nussbaum, Martin and Chaffin [9]. The procedures for this are depicted in Figure 4. What appears to remain for the future is to use ANN technology to study multi-joint, complex reaching motions. Because of the large degree of co-contraction inherent to the shoulder muscles, and the relatively light muscle loads required in a reaching motion, such research will require very sophisticated EMG measurement systems and ANN modeling procedures.
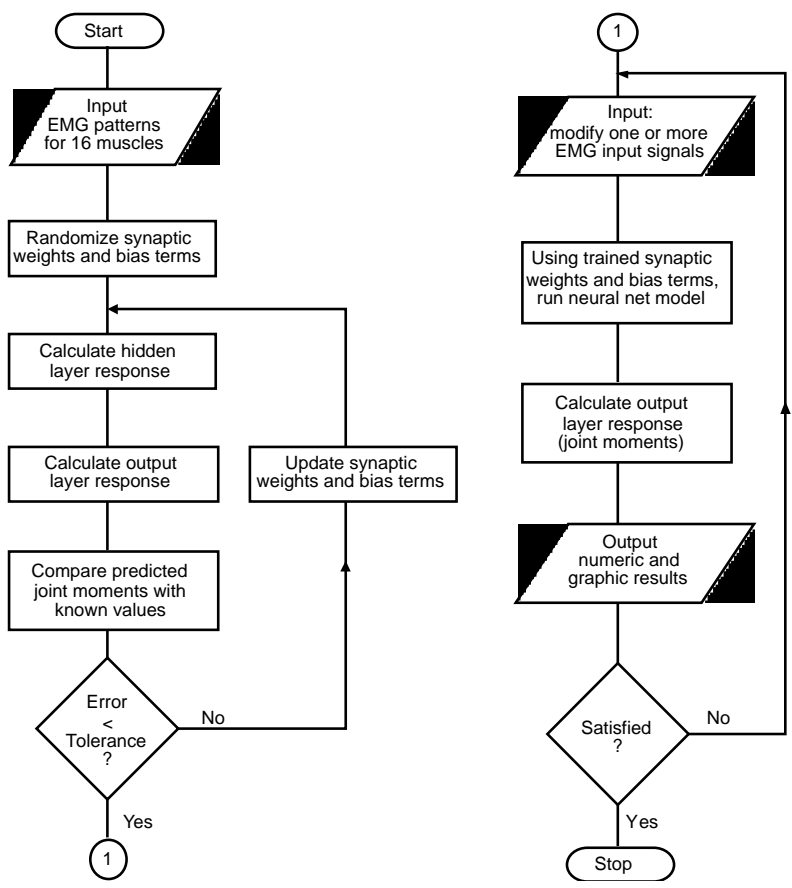

Figure 4. The back-propagation algorithm used to train an ANN may be illustrated in flow chart form (left-hand side), while the actual running of the network is shown on the right [8].

\section{HUMOSIM REACH SIMULATION MODELING}


One of the practical considerations in simulating realistic multi-joint motions is to have efficient software and simple kinematic motion prediction models to enable one to use a common personal computer to perform at least 20 postural predictions per second while still allowing time for updating the rendering of a human graphic form for real-time visualization. This time constraint is especially important for virtual reality applications, as discussed by Badler, Phillips, and Webber [10]. It also is important to provide a visually smooth representation of a simulated motion quickly during workplace and vehicle design analysis exercises to allow "what if" questions to be evaluated efficiently.

The use of inverse kinematic algorithms for sequential static posture prediction have been used for over 20 years in this context. These algorithms typically deal with a kinematic relationship in the displacement domain (e.g., joint angles as a function of hand trajectories). When combined with joint range-of-motion constraints and an objective function, like minimizing the energy or relative strength loading at each joint, nonlinear optimization algorithms can produce a set of sequential postures [10] in a reasonable time frame, though empirical data supporting the fidelity of the resulting postures is not very evident in the literature [11].

\subsection{WEIGHTED PSEUDOINVERSE METHOD FOR} REACH MODELING- Zhang et al. [11] proposed and validated an efficient differential inverse kinematic approach for reach simulation. A four-segment 7-DOF linkage as shown in Figure 5 was used to represent the torso and right upper extremity.

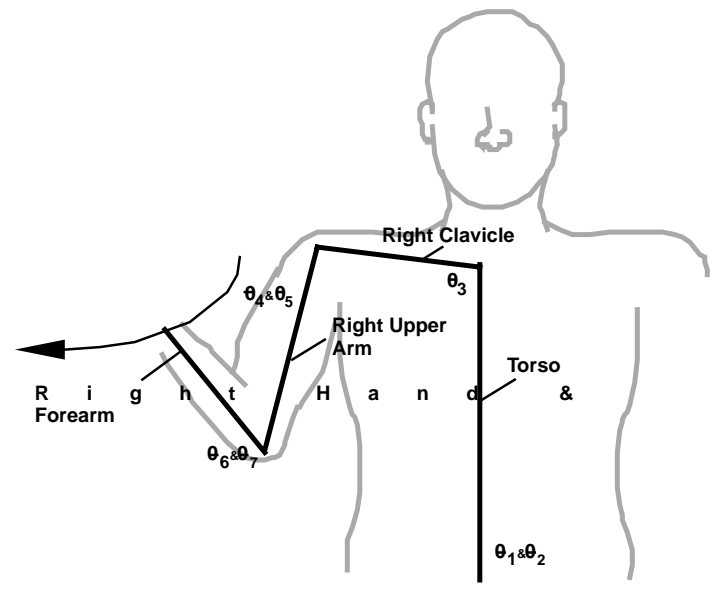

Figure 5. A four-segment seven-DOF linkage representation of the torso and right arm [11].

In this structure, seven degrees of freedom account for the major motions involved in a right-handed seated reach. They are measured by the following seven-joint angles:

$$
\begin{aligned}
& \theta_{1}-- \text { torso flexion; } \\
& \theta_{2}-- \text { torso lateral bending; }
\end{aligned}
$$

$\theta_{3}$--torso twisting (clavicle rotation);

$\theta_{4}$--shoulder extension;

$\theta_{5}$--shoulder abduction;

$\theta_{6}$--humeral rotation; and

$\theta_{7}$--elbow extension.

Based on the above established linkage, the hand position can be derived as a function of the joint angles and link lengths. Assume the link parameters are constant. Let $P=\left[\begin{array}{lll}x & y & z\end{array}\right]^{\top}$ and $\Theta=\left[\theta_{1} \ldots \theta_{7}\right]^{\top}$ be the threedimensional hand position and seven joint angles respectively. The non-linear, complex relationship between $\mathrm{P}$ and $\Theta$ can be expressed in an abstract form as

$$
\mathrm{P}=\left[f_{1}\left(\theta_{1}, \ldots, \theta_{7}\right) f_{2}\left(\theta_{1}, \ldots, \theta_{7}\right) f_{3}\left(\theta_{1}, \ldots, \theta_{7}\right)\right]^{\top}=f(\Theta)
$$

Given the hand position $\mathrm{P}$, a unique posture that has to be fully described by $\Theta$ cannot be determined from the above relationship, because the number of unknowns exceeds the number of equations available (i.e., the system has kinematic redundancy). With the aid of optimization, determination of $\Theta$ is possible but often associated with great complexity, particularly when the $\Theta$ is of large scale. In fact, this is how kinematic redundancy is typically resolved in static optimization approaches for postural modeling.

Differentiating (1) with respect to time results in

$$
\dot{\mathrm{P}}=\dot{f}(\Theta)=\frac{\partial t}{\partial \Theta} \dot{\Theta}=\mathrm{J}(\Theta) \dot{\Theta}
$$

where $\dot{\mathrm{P}}^{\mathrm{Y}}$ is the hand velocity, $\Theta$ represents the joint angular velocities, and $\mathrm{J}$ is the Jacobian matrix mapping $\Theta$ to $\mathrm{P}^{\mathrm{P}}$. For the specific case in consideration, $\mathrm{J}$ is a $3 \times 7$ matrix with each element being

$$
\mathrm{J}_{\mathrm{ij}}=\frac{\partial f_{i}}{\partial \Theta} \quad(i=1,2,3 ; \quad j=1 \ldots 7) .
$$

Equation (2) represents a linear relationship between the hand (end-effector) velocity and joint angular velocity. This method of using the velocity or rate rather than position takes advantage of their linearity and is termed differential kinematics. For redundant systems, there are still an infinite number of $\Theta$ that can provide the same $\mathrm{P}$. However, by hypothesizing an objective function to minimize the weighted Euclidean norm of angular velocity

$$
\mathrm{C}=\|\mathrm{w} \Theta\|
$$

$\Theta$ can be conveniently derived as

$$
\dot{\Theta}=\mathrm{W}^{-1}\left[\mathrm{JW}^{-1}\right]^{\#} \dot{\mathrm{P}} \text {. }
$$


In equation (5), the \# symbolized the pseudoinverse of a matrix, and $\mathrm{W}$ is a symmetric and positive definite $7 \times 7$ weighting matrix which can be expressed as

$$
\mathrm{W}=\mathrm{I} \bullet\left[w_{1} \ldots w_{7}\right]
$$

Where I denotes a $7 \times 7$ identity matrix. Each $w_{i}(i=1-7)$ corresponds to an individual joint angle.

The objective function as expressed by equation (4) can be considered as a measure of the instantaneous weighted effort. Therefore, the weighting parameters $w_{1-7}$ characterize a strategy of allocating the effort among involved joints by minimizing the total weighted effort. A relatively smaller value of the weighting parameter indicates that the corresponding joint angle tends to contribute more in a movement while a greater value would "penalize" any change in the designated angle. Unfortunately the estimation of values for weighting the importance of each joint's motion is not straightforward. A simulated annealing method was developed to derive the approximate values for $\mathrm{W}$ using three error criteria: E1 the absolute angular error averaged across the entire movement and seven joint angles, E2 the absolute angular velocity averaged across the entire movement and seven joints angles, and E3 the absolute angular error of the ending frame averaged across the seven joint angles. A set of 72 different reaching motions performed by six subjects were used to estimate the weights. Each subject's 3D motions were captured at $25 \mathrm{~Hz}$. The motions were chosen to represent a large array of common reaches from near the front of the body to overhead, away from the body and to the right side, The resulting model disclosed reasonable fitting errors could be achieved as shown in Table 1.

Table 1. Statistical summary of fitting errors that result from applying the proposed approach with four time-invariant weighting parameters (one weight designated to each segment) to model 72 seated reach motions.

\begin{tabular}{lccc}
\hline Eval. Function & Mean & Std. Dev. & Median \\
\hline E1 (deg) & 3.3 & 2.7 & 2.4 \\
E2 (deg/frame) & 0.57 & 0.38 & 0.45 \\
E3 (deg) & 4.8 & 4.3 & 4.3 \\
\hline
\end{tabular}

It was also revealed that the weighting values tended to be small for the forearm and arm motions, larger for clavicle segment, and still larger for torso motions.

Thus it was concluded that if a hand trajectory can be defined by a designer who is using this method, the resulting reach postures can be well modeled. Further work is needed, however to extend this approach for a much larger array of one and two handed reaches, and for more complex linkages.
2.2 FUNCTIONAL REGRESSION METHOD FOR REACH MODELING - If one does not wish to specify the intended hand motion trajectory, but rather has only a starting posture and an ending target location to which the hand is to reach, then a more general approach is required. As discussed earlier, inverse kinematics with optimization criteria can be used, but are computationally difficult (time consuming) and may not result in appropriate motions because the optimization criteria that guide coordinate movements would appear to be very dependent on specific situations.

It is for these reasons that we have chosen to develop a very efficient empirical method. It is referred to as the functional regression method for predicting joint angles during the motion of a hand to a specific target. It is particularly useful in dynamic motion modeling wherein 3D motion capture technologies can rapidly acquire very large and dense data sets that are not very noisy throughout a motion (i.e., the joint angles $\theta(t)$ are smooth, regular and known).

Faraway [12] has developed a functional regression model for this purpose. It uses the form:

$$
\begin{aligned}
\theta & (\mathrm{t})=\beta_{\mathrm{o}}(\mathrm{t})+\mathrm{C}_{\chi} \beta_{\chi}(\mathrm{t})+\mathrm{C}_{\mathrm{y}} \beta_{\mathrm{y}}(\mathrm{t})+\mathrm{C}_{z} \beta_{z}(\mathrm{t}) \\
& +\mathrm{C}_{\chi} \mathrm{C}_{y} \beta_{\chi y}(\mathrm{t})+\mathrm{C}_{y} \mathrm{C}_{z} \beta_{y z}(\mathrm{t})+\mathrm{C}_{z} \mathrm{C}_{\chi} \beta_{z \chi}(\mathrm{t}) \\
& +\mathrm{C}_{\chi}{ }^{2} \beta_{\chi}{ }^{2}(\mathrm{t})+\mathrm{C}_{\mathrm{y}}{ }^{2} \beta_{\mathrm{y}}{ }^{2}(\mathrm{t})+\mathrm{C}_{z}{ }^{2} \beta_{z}{ }^{2}(\mathrm{t})+\mathrm{D}
\end{aligned}
$$

where:

$\theta(\mathrm{t})$ are the predicted joint angles over time, $\mathrm{C}_{\chi}, \mathrm{C}_{y}$, and $\mathrm{C}_{z}$ are target coordinates,

$\beta(\mathrm{t})$ are parametric functions to be estimated, and $\mathrm{D}$ are demographic variables (e.g., age, stature, gender, etc) which could modify the predictions.

Note that the model shown is a quadratic model, which was found by Faraway [12] to account for approximately $80 \%$ of the joint angle deviations measured in one set of reach data.

One of the nice aspects of this type of modeling is that eigenfunctions can be derived which provide a means to measure how well a particular model fits a set of data at various points during a motion. It also provides a means to place confidence limits around the $\theta(\mathrm{t})$ 
predictions so that data from different experimental conditions and populations can be statistically compared. An example of this is shown in Figure 6, which shows the elbow included angles for a group of subjects reaching from the steering wheel to an area at the far right side of a radio located in the center of the

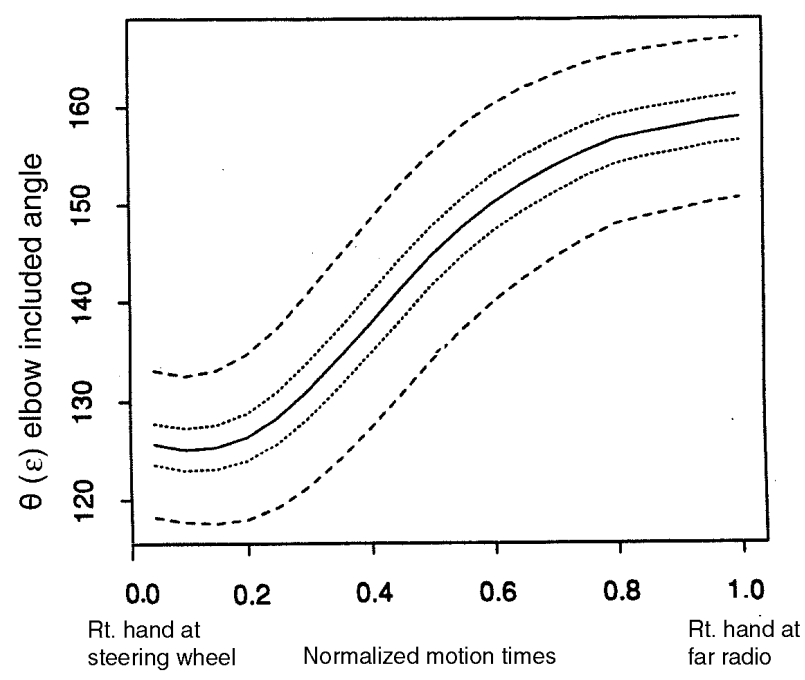

instrument panel.

Figure 6. The predicted elbow angle response (solid line) for a reach to a new target situated between the radio and the glovebox, with $95 \%$ simultaneous confidence bands for the mean response (inner pair) and for the new observation (outer pair) also shown [12].

One of the limitations in this particular empirical approach is that it models the posture only in terms of angles so that when the terminal posture is reconstructed using conventional forward kinematics the hand may not coincide with the reach target. This is due to variation in individual link lengths, the necessarily approximate nature of the model and the variation in reaching behavior. This problem has been attacked by adding an inverse kinematic rectification scheme to the empirical position predictions [13]. The present version resolves the usual redundancy in inverse kinematic predictions by choosing the posture that comes as close as possible to angle-only model predictions while satisfying the hand on the target constraint. Closeness is defined by a metric that takes account of the varying accuracies among the angles that define the posture. This rectification scheme ensures that the hand is on the target in the final posture but it necessarily involves changes in the originally predicted locations of the elbow and shoulder. These changes are usually improvements as demonstrated in [13]. The amount of improvement to be expected depends on many things but in a couple of typical cases, the error at the shoulder was reduced by an average $20 \%$ and the elbow by an average $50 \%$.

A further improvement in the angle predictions is being considered by our group. This method performs the rectification by minimizing the Euclidean distances between the angular velocity trajectories of the empirically predicted and the rectified motion. Preliminary results indicate that such a procedure produces smooth motions (no discontinuities), is computationally efficient and assures the hand ends at the intended target.

\section{HUMOSIM BIOMECHANICS RESEARCH}

The procedures described above provide a means to efficiently estimate values for the joint kinematics involved in normal reaching behaviors. These kinematic values, when combined with a good digital human model provide an improved means of assessing population fit/clearance, reach and visibility requirements.

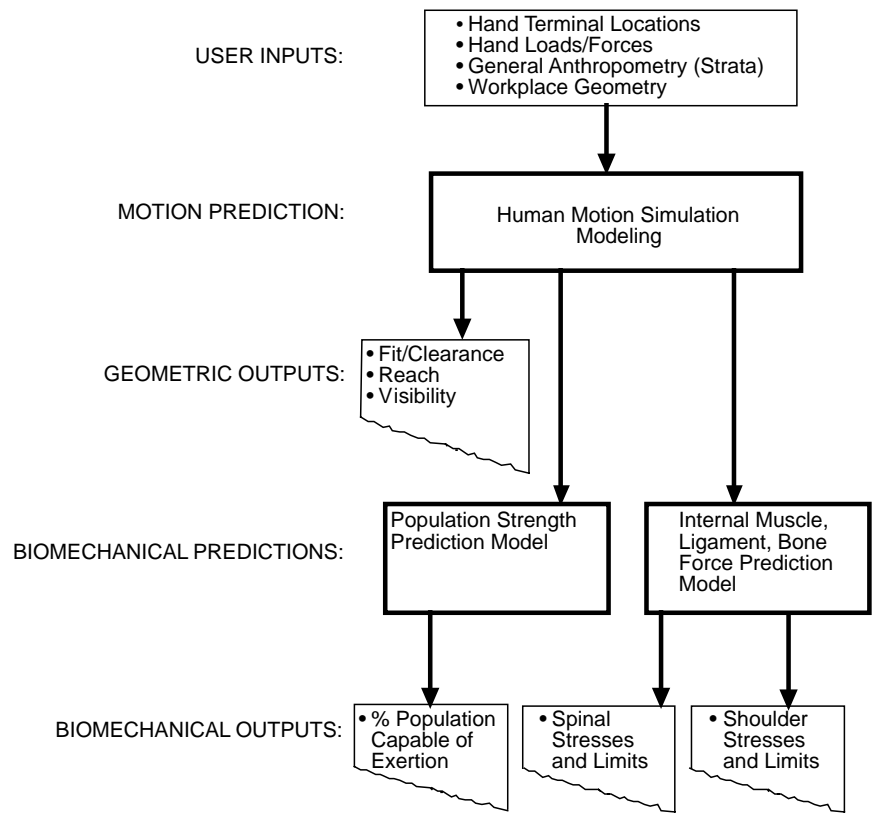

Figure 7. Diagram of University of Michigan's HUMOSIM software project.

In addition, within the Human Motion Simulation HUMOSIM Laboratory we have linked an existing biomechanical model to the kinematic model. The model presently linked is the University of Michigan 3D Static Strength Prediction Program ${ }^{\mathrm{TM}}$. This provides a prediction of population muscle strength requirements at each joint throughout the movement. In addition, estimates of lumbar motion segment compression and shear forces are developed. The joint strength requirements and spinal compression forces can then be compared to NIOSH limits for injury risk analysis. The general logic for these combined programs is illustrated in Figure 7 . We also have been working with a shoulder biomechanical model developed at the University of Gothenberg, Sweden [14]. This model has been used to perform off-line analysis of many different reaching motions by assuring $\mathrm{I} / \mathrm{O}$ compatibility of the motion model and the shoulder model.

\section{HUMOSIM LABORATORY STUDIES}

To develop a robust understanding and database for unweighted and weighted reach modeling we have 
undertaken the following laboratory study. The motions of the head, shoulder, right arm, forearm/hand, and torso segments were first measured during controlled reaches with a group of 20 men and women acting as subjects, We also have enlarged the kinematic model to a sevenlink kinematic model with 19 DOF. This required a more sophisticated motion capture system, which now employs both an electro-magnetic (EM) Flock of Birds system integrated with an optical Qualysis Expert Vision system. The linkage and sensor locations are depicted in Figure 8.

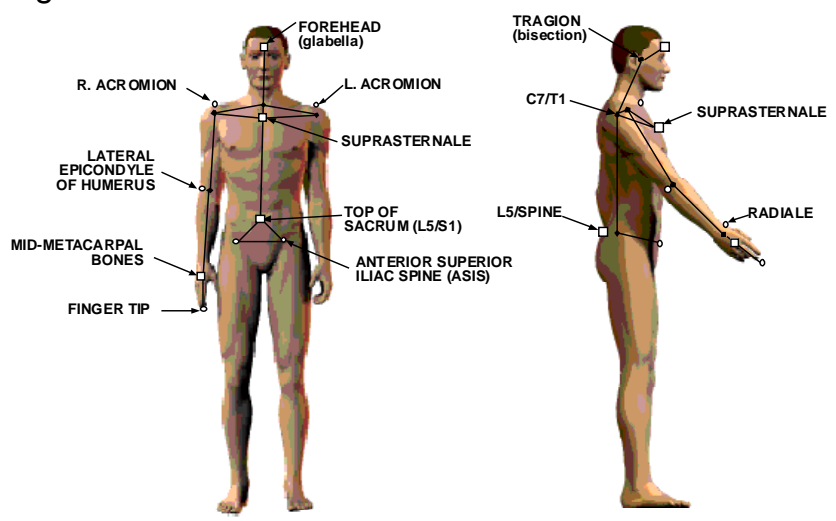

Figure 8. The internal kinematic linkage and surface EM (凶) and optical $(O)$ markers used in HUMOSIM seated reach modeling.

A scheme for palpating 11 well defined bony landmarks on subjects was developed, which provided 19 anthropometric variables (plus age and shoulder strengths) to be available for scaling the linkage system. In addition, 6 global and local joint angle coordinate systems were defined to represent the instantaneous positions of the segments comprising the linkage system. The new linkage system has been programmed into EAI-Transom $\mathrm{JACK}^{\mathrm{TM}}$ to compute the joint angles from the motion capture systems.

\subsection{INDUSTRIAL SEATED REACHES - The} industrial seat used in this study places the worker at a level wherein the work bench is normally near elbow height for an average size male when sitting erect. This results in an $\mathrm{H}$ point about $67 \mathrm{~cm}$ above the floor. The industrial seat used in this study had a lumbar support and narrow backrest, which would allow considerable torso rotation.

A small visual display was positioned on a shelf in front of the seated person, slightly above erect elbow height. This was meant to simulate the visual requirement of a seated manufacturing/assembly operation. The right and left hands rested immediately in front of the display, with the right index finger resting on a contact switch, to record the initiation and completion of each reaching motion.

Figure 9. Diagram of targets in industrial reach simulator - front targets move in and out.

Fifty-two (52) targets were positioned around the subject. As shown in Figure 9, the targets were chosen in this case to simulate different reaches across a large area in front of the person, as well as to overhead, to far right side, and to near right side floor level.

After a subject had completed a set of no-load motions, a hand load was strapped in the palm of the hand, the magnitude of which depended on the person's shoulder strengths. The reaches were then repeated with the hand load.

\subsection{AUTOMOBILE SEATED REACHES - Since the} earlier automotive database was acquired with a relatively deep seat back and seat pan bolsters, both of which could alter some of the reaching postures, it was decided to perform the new studies with a seat having a less intrusive bolster design, common to many lower priced vehicles. The same type of reaches as used in the industrial reach study were used, but the visual display was mounted forward of the person and a steering wheel and pedal set was added to the fixture.

\subsection{TRUCK/BUS SEATED REACHES - Since a}

truck/bus seat configuration raises the driver's $\mathrm{H}$ point approximately $22 \mathrm{~cm}$ from that in an automobile $(\mathrm{H}$ point $=$ $26 \mathrm{~cm}$ ), and normally may be quite flat to allow easier access and mobility, a separate study was initiated using a typical light truck/bus seat. The same set of reach targets used for the automobile seat were used in this study.

\section{SUMMARY}

This paper has described several different approaches now being used to study and understand human reach behaviors. The philosophy adopted within the HUMOSIM Laboratory has been one that assures that any kinematic models used to predict dynamic reaches are representative of actual movements performed in a variety of circumstances. We have proposed that a combination of new functional regression and optimization based inverse kinematic models can be used to meet this objective.

We also propose that once the kinematics of certain reaches are successfully modeled, the resulting motion parameters can be used as inputs to existing and future biomechanical models, thus providing an excellent means to assess ergonomic features within future digital mock-ups. 
As a side benefit of this approach, because the motion kinematics of groups of people are being studied and quantified in statistical models, it is possible to delineate the variability in motions between groups of people. For instance, our motion simulation models now reflect movement pattern differences due to gender and age as well as anthropometry. A new study underway will determine if a special type of motion aberration is associated with people suffering from chronic mental depression. Eventually, it is believed that the biomechanical models now linked to our motion simulation models will allow the prediction of movement limitations and compensation when a person has a specific muscle deficit or weakness, thus assisting in the rehabilitation and return-to-work planning for such individuals.

In summary, advanced motion capture systems, database management methods, and new kinematic and statistical modeling methods provide an excellent basis for dramatically improving human motion simulation technologies. Our HUMOSIM Laboratory is dedicated to this development, and we welcome those interested in this exciting venture.

\section{Acknowledgement}

The authors wish to acknowledge and thank our supporting partners in the HUMOSIM Laboratory, Ford Motor Company, General Motors, Navistar, TRW, Johnson Controls, and the Daimler-Chrysler Challenge Fund, as well as our colleagues Charles Woolley, James Foulke, Woojin Park, and Yao Wang.

\section{REFERENCES}

[1] Latash, M.L. (1998). Neurophysiological Basis of Movement, Human Kinetics, Champaign, IL.

[2] Gielen S., van Bolhois, B., and Vrijenoek, E. (1998). On the Number of Degrees of Freedom in Biological Limbs, in M.L. Latash, Progress in Motor Control - Bernstein's Tradition in Movement Studies, Human Kinetics, Champaign, IL.

[3] Barnes, S., Oggero, E., Pagnocco, G. and Berme, N. (1998). Simulation of Human Motion, in , P. Allar, A. Cappozzo, A. Lundberg, and C. Vaughn (Eds.), Three-Dimensional Analysis of Human Locomotion, Wiley, New York.

[4] Winters, J.M. (1995). How Detailed Should Muscle Models be to Understand Multi-joint Movement Coordination, Human Movement Science, 14:401442.

[5] Zajac, F., and Winters, J.M. (1990). Modeling Musculoskeletal Movement Systems, in J.M. Winters and S.L. Woo (Eds.), Multiple Muscle Systems Springer Verlag, New York.
[6] Feldman, A.G., and Levin, M.F. (1995). The Origin and Use of Positional Frames in Reference Motor Control, Behavioral and Brain Sciences, 18:723-806.

[7] Massion, T.J., Alexandrov A., and Vernazza, S. (1998). Coordinated Control of Posture and Movement, in M. Latash (Ed.), Progress in Motor Control, Human Kinetics, Champaign, IL.

[8] Vaughn, C. (1998). Neural Network Models of the Locomotion Apparatus, in P. Allard A. Cappozzo, A. Lundberg, and C. Vaughn (Eds.), Three Dimensional Analysis of Human Locomotion, Wiley, New York.

[9] Nussbaum, M., Martin B., and Chaffin, D. (1997). A Neural Network for Simulation of Torso Muscle Coordination, Journal of Biomechanics 30(3):251258.

[10] Badler, N., Phillips, C., and Webber, B. (1993). Simulating Humans, Oxford University Press, New York.

[11] Zhang, X., Kuo, A.D., and Chaffin, D.B. (1998). Optimization based Differential Kinematic Modeling Exhibits a Velocity-control Strategy for Dynamic Posture Determination in Seated Reaching Movements, Journal of Biomechanics 31:10351042.

[12] Faraway, J. (1997). Regression Analysis for a Functional Response, Technometrics 39(3):254-262.

[13] Faraway, J., Zhang, X., and Chaffin, D. (1998). Rectifying Postures Reconstructed from Joint Angles to meet Constraints, Journal of Biomechanics (Submitted).

[14] Karlsson, D., and Peterson, B. (1992). Towards a Model for Force Predictions in the Human Shoulder, Journal of Biomechanics, 25(2):189-199.

* For correspondence please contact: Professor Don Chaffin, Center for Ergonomics, 1656 Industrial and Operations Engineering Building, The University of Michigan, Ann Arbor, MI 48109-2117, USA, phone: 734-763-2245, fax 734-764-3451, email: dchaffin@engin.umich.edu 